\title{
Influence of DC Magnetic Field on AC Loss of YBCO Coated Conductor with Ferromagnetic Substrate
}

\author{
M. VojenčIak, J. Šouc, F. Gömöry and E. Seiler \\ Institute of Electrical Engineering, Centre of Excellence CENG \\ Slovak Academy of Sciences \\ Dúbravská cesta 9, 84104 Bratislava, Slovakia
}

\begin{abstract}
Most of YBCO tape conductors are prepared on a ferromagnetic substrate. The ferromagnetic material is then exposed to the self magnetic field of the superconductor and also to external magnetic field. In AC magnetic field hysteretic and eddy current losses are generated in the substrate. In this work the AC transport loss of an YBCO coated conductor on a ferromagnetic substrate was measured using electromagnetic method. There are two contributions to total loss - a loss in the superconductor and a loss in the substrate. To suppress the hysteretic loss in the substrate, it is possible to saturate it using DC magnetic field. In this regime significant decrease in total loss was observed.
\end{abstract}

PACS numbers: 74.25.Fy, 74.25.Nf, 74.78.Bz, 75.50.-y

\section{Introduction}

Long length YBCO tape superconductors are made using ferromagnetic substrates. Although some of these materials has low Curie temperature, all of them are ferromagnetic below critical temperature of an YBCO [1].

The YBCO coated conductor is often modeled using model of infinitely thin strip [2]. This model predicts lower AC loss than model assuming elliptical superconductor cross-section (case of BiSCCO tape). This was a reason to expect lower AC loss of YBCO tapes in comparison to BiSCCO ones. Measurements of the AC losses due to transport currents show higher loss than theoretical works predict $[3,4]$. This may be caused by several reasons - inhomogeneity of YBCO layer, interaction between superconductor and ferromagnetic substrate, and by contribution of hysteretic losses in the substrate to total loss of the tape. We suppress the last mentioned contribution using DC magnetic field parallel with wide face of the sample. Relatively low external DC magnetic field saturates the substrate magnetization without a significant critical current decrease. In this regime the substrates magnetization has two contributions - DC one caused by external 
magnetic field and $\mathrm{AC}$ one caused by the self-field of the superconductor. As a consequence, hysteretic loss decreases when DC part of magnetization increases.

\section{Experimental setup}

The AC loss was measured using electromagnetic method. YBCO coated superconducting tape carrying AC transport current was subjected to the DC external magnetic field oriented parallel to the wide face of the sample.YBCO coated superconducting tape carrying AC transport current was subjected to the DC external magnetic field oriented parallel to the wide face of the sample. Dependence of $\mathrm{AC}$ losses on $\mathrm{AC}$ transport current was measured. In each measurement a constant level of DC external magnetic field was kept.

In this work the YBCO coated tape prepared on a ferromagnetic substrate with critical current $81 \mathrm{~A}$ was examined. $10 \mathrm{~cm}$ long tape was placed into race track shaped magnet generating DC magnetic field.

In order to avoid flowing of $\mathrm{AC}$ current in magnet due to a mutual inductance of DC and $\mathrm{AC}$ circuits, the inductance of $100 \mathrm{mH}$ was added to the DC circuit. The absence of the coupling was checked by measuring of the $\mathrm{AC}$ current in the magnet circuit. This current was less than $0.1 \%$ of the DC current.

\section{Results and discussion}

In Fig. 1 the dependence of the transport $\mathrm{AC}$ loss on the root mean square (rms) value of transport current at different DC magnetic fields, measured at frequency of $72 \mathrm{~Hz}$, is shown. Dash-dotted and dashed curves represent the prediction obtained using the Norris model for superconductor cross-section with elliptical and infinitely thin strip shape, respectively. The measured loss is higher than theoretical models predict.

The total loss at magnetic fields lower than $7 \mathrm{mT}$ are lower than self-field transport loss. This is caused by decrease in hysteretic losses in substrate. At magnetic fields higher than $7 \mathrm{mT}$ an increase in the total loss was observed. This may be caused by decrease in critical current.

Slope of all the measured curves is lower than is expected for a superconductor in a self-field. This observation indicates that besides the loss in superconductor there is another loss, which increases less than that in superconductor.

In Fig. 2 the dependence of the total loss of the tape on parallel DC magnetic field is shown. There are two groups of curves, each for one value of the transport current. From this representation it is possible to see the influence of external DC magnetic field.

The loss was measured at four frequencies. Interesting result is that the loss is lower at higher frequencies and the same magnetic field, especially at higher fields. This tendency is the same in the whole range of measurements. Minimum loss for the same frequency and various transport currents is at a higher external magnetic field for a higher transport current. Higher transport current causes 


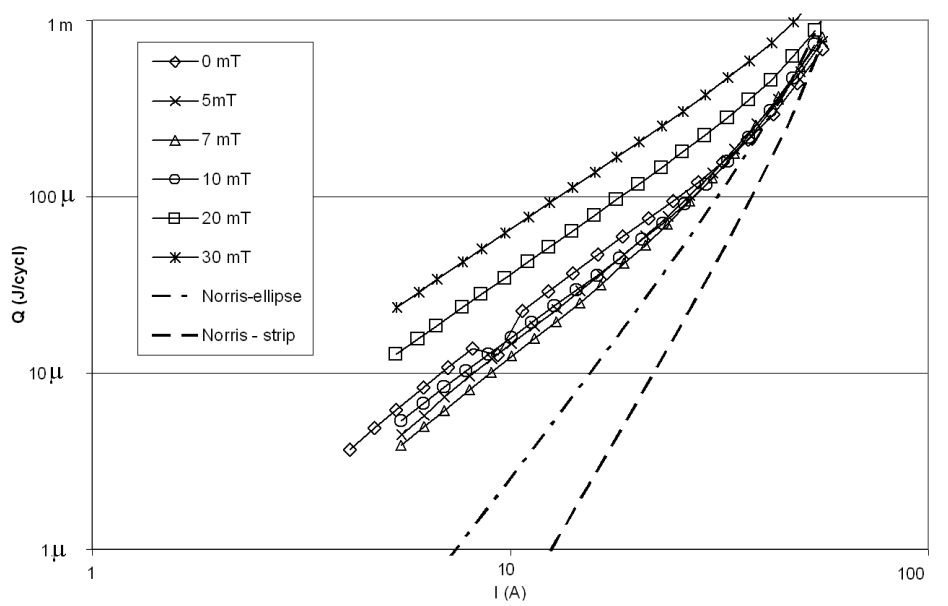

Fig. 1. Measured dependences of the tape total loss on the rms transport current value in various values of DC magnetic field parallel to the tape plane.

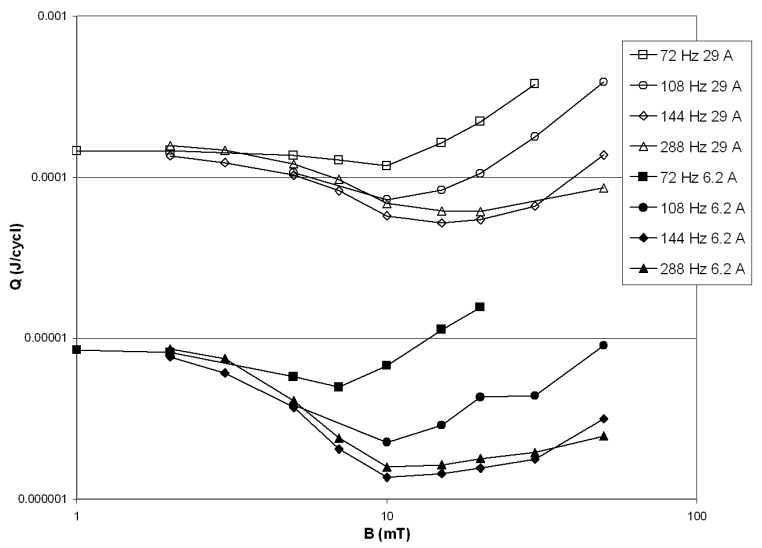

Fig. 2. Transport loss at a constant AC transport current as a function of an applied DC magnetic field parallel to the tape plane.

higher self-field and therefore a higher DC external field is needed to saturate the magnetization in the substrate.

\section{Acknowledgments}

This work was partially supported by the Slovak Research and Development Agency (contract APVV 51-045605). 


\section{References}

[1] A.O. Ijaduola, J.R. Thompson, A. Goyal, C.L.H. Thieme, K. Marken, Physica C 403, 163 (2004).

[2] W.T. Norris, J. Phys. D 3, 489 (1970).

[3] J. Ogawa, H. Nakayama, S. Odaka, O. Tsukamoto, Physica C 412, 1021 (2004).

[4] J. Šouc, M. Vojenčiak, F. Gömöry, X. Granados, A. Usoskin, A. Rutt, J. Phys. Conf. Ser. 43, 127 (2006). 